\title{
INTRODUCTION
}

\section{The Medical Insurance for a New Generation: another step towards universal health coverage in Mexico}

$\mathrm{T}^{\mathrm{h}}$ The Medical Insurance for a New Generation (Seguro Médico para una Nueva Generación, SMNG) is a novel program within the System of Social Protection for Health, most commonly known as Seguro Popular. It is a tax-based social health insurance scheme and its aim is to protect children under five years old who are not covered by social security from financial and health burden by facilitating access to healthcare.

Gaps in equity, access, coverage and health status of Mexican children fueled the decision of the Mexican government to launch the program. Seguro Popular is committed to developing a high profile agenda of evaluative studies upon which to build the knowledge and institutional capability to reach the goals of universal health coverage. Following this policy, Seguro Popular chose Hospital Infantil de México Federico Gómez to run the first evaluation of SMNG. This evaluation took place in 2009 and engaged researchers from different disciplines and decision-makers to obtain a comprehensive and unbiased picture of SMNG and its members.

The evaluation was focused on five domains: 1) the design of the program; 2) the social and health conditions of its members; 3 ) access and use of health services; 4) performance through measuring coverage and key processes; and 5) the effect of SMNG on families' health expenditures.

The articles of this series show the results from different perspectives that include the characteristics of the population, the current situation of the program and the quality and performance of the providers.

The status of Mexican children is analyzed in-depth, stressing the presence of unmet health needs, such as anemia and malnutrition and the growing problem of obesity among children. The analysis also provides a brief picture of the persistence of inequities in this age group and the need to implement the program to decrease the toll of infant deaths and alleviate the burden of families to care for their sick children.

The 2009 SMNG evaluation used a mixed multimethod approach. A household survey with national and urban-rural representativeness was conducted. Hospital Infantil de México partnered with Instituto Nacional de Estadistica y Geografía (INEGI) to run the survey. Information from the registries of SMNG and from the Fund for Protection against Catastrophic Expenditures (Fondo de Proteccion contra Gastos Catastróficos) was also reviewed. Information from primary sources included review of clinical charts, interviews with medical doctors and directors of primary, secondary and tertiary care facilities. The information obtained from the household survey served to analyze several aspects related to SMNG members in depth, such as their current socio-demographic characteristics, which included the composition of the household and their living conditions, their feeding practices and nutritional status, the relationship between the socioeconomic factors and anemia, and current patterns of utilization of preventive and curative health care. Information about mortality among SMNG members was obtained from the household survey. This at-a-glance information of SMNG member mortality provides a good indicator about the magnitude and importance of this problem.

The evaluation of performance through measuring coverage and key processes was conducted addressing different evaluative perspectives: characteristics of the enrollment process, availability of SMNG and FPGC information, fulfillment of the indicators defined in the operating rules. Furthermore, the evaluation of this com- 
ponent addressed the quality of structure and processes in primary, secondary and tertiary care facilities, and used the tracer methodology to assess quality of care in four problems: low birth weight, iron supplementation, congenital hypothyroidism, and respiratory distress syndrome. In this same direction, a thorough evaluation of care for newborns with respiratory distress syndrome was conducted in a sample of tertiary care hospitals with neonatal intensive care units. The evaluation defined a set of recommendations to improve care for these children, which accounted for most neonatal mortality rates. In this sense, the economic evaluation of the use of exogenous pulmonary surfactants in preterm newborns with respiratory distress syndrome was evaluated, showing that this medication is cost-effective.

An important goal of SMNG is to mitigate the economic burden of families to care for their sick children. An evaluation of the out-of-pocket and catastrophic health expenditures was conducted using the information from the household survey. It shows that more needs to be done to decrease the hardship of families.

The evaluation shows strengths and weaknesses of the program and the aim of Seguro Popular is to dis- seminate these findings to the different stakeholders, the public, decentralized Ministry of Health in the states and clinicians staffing primary, secondary and tertiary care facilities and to those interested in the health of children. It is expected that the benefits of SMNG will accrue and this evaluation sets the groundwork to establish better subsequent interventions and evaluations. The evaluation shows that SMNG is strengthening its role as a key stakeholder of Mexican policies aimed at improving the health of children. The need to build the capacity of primary care clinics and secondary care hospitals was clearly identified and this is a necessary step to be able to provide high quality care. Fulfilling the programmatic goals of coverage is not enough. It is also necessary to implement measures to improve quality and ease the economic burden of the families. The program has the capacity to contribute to reaching the goal of universal health coverage for children, however, health providers must work harder to be able to show the improvement of the health status of Mexican children.

Salomón Chertorivski Woldenberg*

\footnotetext{
* Secretario de Salud, México.
} 